EXTENDED REPORT

\title{
Late onset post-keratoplasty astigmatism in patients with keratoconus
}

\author{
L Lim, K Pesudovs, M Goggin, D J Coster
}

Br J Ophthalmol 2004;88:371-376. doi: 10.1136/bjo.2003.027037

\begin{abstract}
See end of article for authors' affiliations

....................

Correspondence to: Douglas John Coster, Department of Ophthalmology, Flinders Medical Centre, Flinders University of South Australia, Bedford Park 5042, South Australia; doug.coster@fmc.sa. gov.au

Accepted for publication 1 August 2003
\end{abstract}

\begin{abstract}
Aim: 10 eyes of 10 patients are reported where progression of keratoconus in the host cornea occurred more than 10 years after penetrating keratoplasty with resultant increase in astigmatism. The technique and results of graft refractive surgery in seven eyes are presented.

Methods: The clinical features and management of these patients were retrospectively analysed. Graft refractive surgery involved an incision at the graft-host junction adjacent to the host thinning with compressive resuturing. Astigmatic changes were calculated using vector analysis.

Results: There were seven men and three women with a mean age of 41.2 years. The average age when undergoing penetrating keratoplasty in the affected eye was 28.4 years and the average time after penetrating keratoplasty until keratoconus appeared in the host cornea defined by host thinning was 13.5 years. The mean cylinder power before host thinning was noted was 5.07D (SD 2.19) and the mean after host thinning was 11.0D (2.53). The mean vector calculated disease induced astigmatism magnitude was 7.59D (3.09). Graft refractive surgery was performed in seven eyes. The mean cylinder power before and after graft refractive surgery was 11.28D (2.15) and 7.09D (5.53) respectively. The surgically induced astigmatism vector magnitude was 7.36D (4.88).

Conclusion: Progression of keratoconus in the host cornea late after penetrating keratoplasty is characterised by a large astigmatic change where the flat axis of astigmatism passes through an area of host thinning visible on slit lamp examination. Compressive resuturing performed in the area of host thinning resulted in satisfactory reduction of astigmatism.
\end{abstract}

$\mathrm{K}$ eratoconus is a progressive, non-inflammatory condition in which the central cornea becomes increasingly ectatic with time. The disease typically manifests in the second or third decade of the patient's life. Penetrating keratoplasty (PK) is indicated when vision is not satisfactory with glasses or contact lenses. Penetrating graft survival rates range between $94 \%$ to $97 \%$ at 5 years after grafting ${ }^{12}$ and corrected visual acuity of 6/12 or better are obtained in 86$91 \%$ of eyes. ${ }^{23}$

Astigmatism is common following corneal transplantation and it is a particular problem in PK performed for conditions associated with astigmatism such as keratoconus.

Recurrence of keratoconus in the donor tissue after penetrating keratoplasty has previously been described ${ }^{4-8}$ with histological confirmation in some cases. ${ }^{4578}$ The mean latency of the reported keratoconus cases is 17 years, which is analogous to the natural progression of keratoconus in adolescence.

Although keratoconus may be confined to the central cornea initially, it may progress to involve the peripheral cornea. Progression of keratoconus in the host cornea after PK has also been known to occur. ${ }^{9}$ Penetrating keratoplasty may replace the affected cornea at the time of surgery, but keratoconus (thinning and ectasia) in the host peripheral cornea can occur and seems to arrive at the host cornea as if it had gradually extended from the excised central cornea. When this happens it produces a distinct clinical pattern of increased astigmatism with the flat axis through the area of thinning.

In this retrospective review, we report 10 eyes of 10 patients where we believe that keratoconus has progressed into the host cornea with resultant increase in postkeratoplasty astigmatism. We also report the results of graft refractive surgery seven cases and would like to suggest an algorithm for graft refractive surgery in cases with progression of keratoconus in the host cornea.

\section{PATIENTS AND METHODS}

We analysed retrospectively the clinical features and the surgical outcome of 10 eyes in 10 patients who sought treatment from one surgeon (DJC) for progression of keratoconus in the host cornea after PK. The sources of information were the hospital records and, where applicable, the records of ophthalmologists and optometrists who referred these patients. The data collected included patient demographic information, area of the host cornea where progression of keratoconus occurred as confirmed by slit lamp examination, duration after grafting when host thinning occurred, keratometry, refraction, corneal topography, and corrected visual acuity before and after progression of keratoconus in the host cornea and after graft refractive surgery, type, and location of graft refractive surgery, duration before suture removal, and number of sutures remaining after suture removal.

\section{Graft refractive surgery}

Preoperative refraction was difficult to perform in some patients owing to the high astigmatism involved. Although it would be ideal to have preoperative refractions on all these patients, we relied primarily on keratometry in the planning of the surgery. Those who required surgery could not achieve satisfactory vision with contact lenses or spectacles.

Compressive suturing was performed in the quadrant of host thinning which corresponded to the area with the flattest meridian on keratometry. This involved making an incision of approximately $90 \%$ depth at the graft-host junction spanning 2-3 clock hours, on either side of the flattest meridian. This is followed by compressive suturing of the incision with 3-6 interrupted 10-0 Nylon sutures to achieve an overcorrection, which was verified in the operating theatre with the use of an operative keratometer. Selective suture removal was performed a few months later to give the optimum cylindrical correction. 


\section{Vector analysis of astigmatism}

Vector analysis was carried out using the Alpins method, with minor modifications to allow for the analysis of disease induced astigmatism. ${ }^{10}$ Vector values for disease induced astigmatism, targeted induced astigmatism (TIA), and surgically induced astigmatism (SIA) were derived and analysed using keratometric data.

To analyse the astigmatic change brought about by disease progression in the host cornea, the change in keratometric readings was used to calculate a vector value for this change, the disease induced astigmatism. This may be defined as the degree of corneal flattening induced by the disease. However, since we use the plus cylinder convention and analyse astigmatism using the steepest axis the figure derived is the steepening of the meridian at right angles to the meridian affected by the disease. Otherwise the vector analysis of disease induced astigmatism is analogous to surgically induced astigmatism. The target of surgery was to reduce the cylinder power to that which can be more easily managed by contact lenses or spectacles, approximately 4D. All procedures were carried out "on axis"; thus, no attempt was made to alter the cylinder axis from that found immediately preoperatively.

\section{RESULTS}

There were seven men and three women with a mean age of 41.2 years (range $34-45$ years). The average age at undergoing PK in the affected eye was 28.4 years (range 1644 years). The average time after PK during which keratoconus progressed in the host cornea was 13.5 years (range 10-18 years). These patients presented with a decrease in visual acuity associated with increased astigmatism after having previously had more than 10 years of stable refraction after PK. The duration of symptoms was relatively short with a mean of 10.2 months (range 1-24 months). We were unable to obtain accurate data on graft size; we estimate most were between $7.00 \mathrm{~mm}$ to $7.50 \mathrm{~mm}$ in diameter.

On examination of the affected eye, an area of host corneal thinning was seen (fig 1). This corresponded to the flattest meridian on keratometry and was observed in all cases. Host corneal thinning occurred in the nasal quadrant in five eyes, nasal and temporal quadrant in three eyes, superonasal quadrant in one eye, and inferior quadrant in one eye. The graft astigmatism on keratometric measurements before host thinning, after host thinning and the graft astigmatism after refractive surgery are shown in table 1 and figure 2 . The mean cylinder power before thinning of the host was 5.07D (SD 2.19D). The mean after thinning was 11D (2.53D). The mean vector calculated disease induced astigmatism magnitude was 7.59D (3.09D). The change in axis from the

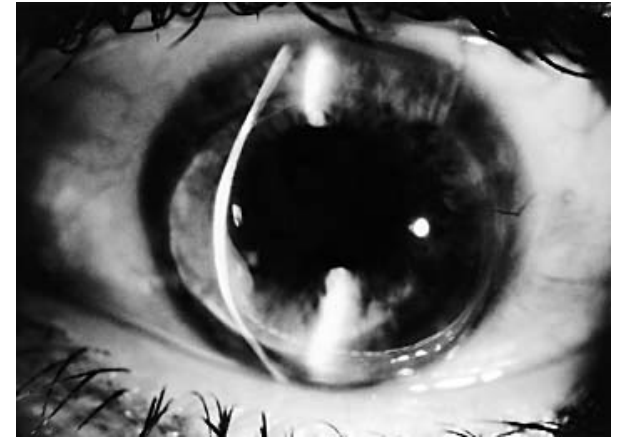

Figure 1 Slit lamp photography showing progression of keratoconus in the host cornea inferiorly resulting in host tissue thinning.

astigmatism vector before thinning to the vector derived after thinning is given a sign depending on the direction of alteration. If the axis changes in an anticlockwise direction, it is given a plus sign and clockwise, a minus sign. The mean axis change was $+21.4^{\circ}\left(55.98^{\circ}\right)$.

Compressive resuturing was performed in seven eyes. Patient 2 had excessive thinning in both the nasal and temporal quadrants and therefore wedge excisions and compressive resuturing were performed in both quadrants. Patient 7 had progression of keratoconus in both the nasal and temporal quadrants hence compressive resuturing was performed in both quadrants. Patient 8 did not have graft refractive surgery, as he was still able to cope with corrective glasses. Patients 9 and 10 had graft refractive surgery less than 3 months earlier and therefore postoperative follow up was not included in the table.

The average time between graft refractive surgery and suture removal was 7.3 months (range 5-15 months). Five eyes had all sutures removed; two eyes had one and two sutures remaining, respectively. The mean follow up after graft refractive surgery was 19.1 months (range 660 months). All patients had significant improvement in their visual acuity and all could wear corrective glasses whereas before surgery correction with glasses or contact lenses was difficult (table 2, fig 3). The mean (SD) astigmatism after graft refractive surgery was 6.78D (5.20). There appears to be little difference in astigmatism before and after graft refractive surgery for patients 3, 5, and 7 . This could be due to a relative earlier removal of sutures for these patients $(6,5$, and 5 months respectively) compared to the average of 7.3 months. In these eyes the surgically induced astigmatism fell well short of the targeted induced astigmatism. However, in patient 3, the SIA exceeds the TIA, in

Table 1 Keratometric cylindrical differences before and after host thinning and after graft refractive surgery (GRS)

\begin{tabular}{|c|c|c|c|c|c|c|}
\hline Patient & $\begin{array}{l}\text { Astigmatism before } \\
\text { host thinning (D) }\end{array}$ & $\begin{array}{l}\text { Astigmatism after } \\
\text { host thinning (D) }\end{array}$ & $\begin{array}{l}\text { Disease induced } \\
\text { astigmatism vector } \\
\text { magnitude }\end{array}$ & $\begin{array}{l}\text { Astigmatism } \\
\text { after GRS (D) }\end{array}$ & $\begin{array}{l}\text { Surgically induced } \\
\text { astigmatism vector } \\
\text { and orientation }\end{array}$ & $\begin{array}{l}\text { Targeted induced } \\
\text { astigmatism vector } \\
\text { magnitude }\end{array}$ \\
\hline 1 & $1.75 \times 100$ & $9.75 \times 90$ & 8.12 & 0.00 & $9.75 \times 180$ & 5.75 \\
\hline 2 & $4.00 \times 122$ & $12.88 \times 100$ & 10.38 & $2.13 \times 160$ & $14.06 \times 6$ & 8.88 \\
\hline 3 & $6.95 \times 30$ & $14.46 \times 60$ & 12.50 & $14.13 \times 38$ & $10.72 \times 3$ & 10.47 \\
\hline 4 & $4.38 \times 2$ & $10.13 \times 55$ & 12.09 & $4.13 \times 20$ & $9.54 \times 157$ & 6.13 \\
\hline 5 & $5.50 \times 85$ & $9.00 \times 95$ & 4.27 & $8.63 \times 93$ & $0.72 \times 33$ & 5.00 \\
\hline 6 & $4.50 \times 12$ & $9.63 \times 15$ & 5.17 & $6.63 \times 10$ & $3.31 \times 115$ & 5.63 \\
\hline 7 & $7.00 \times 110$ & $13.16 \times 115$ & 6.38 & $14 \times 108$ & $3.41 \times 73$ & 9.16 \\
\hline 8 & $5.25 \times 75$ & $8.75 \times 65$ & 4.22 & & & \\
\hline 9 & $9.00 \times 120$ & $14.59 \times 110$ & 6.86 & & & \\
\hline 10 & $2.38 \times 8$ & $7.63 \times 170$ & 5.88 & & & \\
\hline \multirow[t]{2}{*}{ Mean (SD) } & 5.07 (2.19) & $11.00(2.53)$ & 7.59 (3.09) & $7.09(5.53)^{*}$ & $7.36(4.88)$ & $7.28(2.15)$ \\
\hline & & $11.28(2.15)^{*}$ & $8.42(3.32)^{*}$ & & & \\
\hline
\end{tabular}

*Mean of seven eyes with keratometric data before and after graft refractive surgery for comparison. 


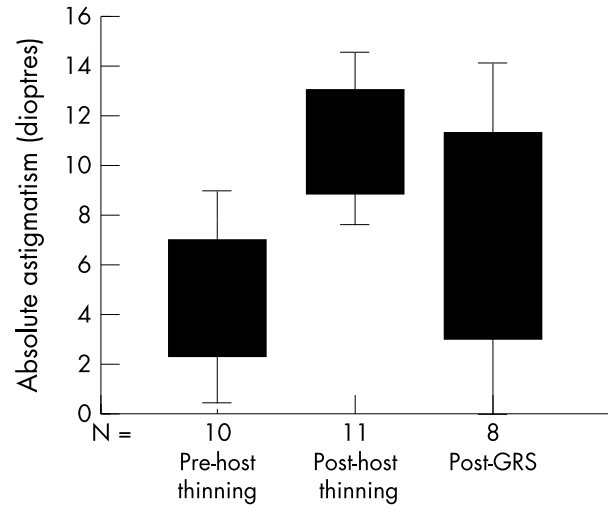

Figure 2 Box plots illustrating graft astigmatism before host thinning, after host thinning, and after graft refractive surgery (GRS).

magnitude, but is off axis by almost 60 degrees leaving a large cylinder. Patient 3 also developed inferior graft fibrosis following compressive suturing, which may have contributed to this off-axis effect. However, on refraction, 6/9 vision was achieved with $-1.75 /-4.00 \times 108^{\circ}$.

Patient 1 developed endothelial graft rejection 1 month after graft refractive surgery but this resolved with topical steroid treatment over 3 months. Figures 4 and 5 are corneal topography maps of patient 2 before and after graft refractive surgery. Figure 4 is a composite map showing the corneal topography before surgery (top left), after surgery but before suture removal (right), and after selective suture removal with two sutures remaining (bottom left). Figure 5 illustrates the effect of wedge excision and compressive resuturing with deliberate initial overcorrection before suture removal.

Seven eyes had keratometric data available before and after refractive surgery for astigmatism. The mean cylinder, preoperatively, was $11.28 \mathrm{D}(2.15)$ and postoperatively was 7.09D (5.53). To achieve $4 \mathrm{D}$ on-axis of remaining astigmatism, the mean TIA vector magnitude was $7.28 \mathrm{D}$ (2.15). Vector analysis of the final keratometric readings demonstrates the SIA vector magnitude was 7.36D (4.88). The mean magnitude of error (that is, the difference between the SIA and TIA) shows a small "overcorrection" of 0.07D (4.3). However, since any astigmatism less than 4D was deemed acceptable, this is only an overcorrection in terms of vector analysis where, to calculate how well the surgery achieved its aim, the target has to be given a specific value. To obtain a residual cylinder value of zero would still be desirable although difficult to achieve.

The angle of error is the difference between the SIA vector angle and the TIA vector angle. It is a measure of the accuracy of the meridional placement of the surgery and its predict-

Table 2 Improvement of visual acuity (VA) after graft refractive surgery

\begin{tabular}{lll}
\hline Case & VA before GRS & VA after GRS \\
\hline 1 & $6 / 12$ (glasses) & $6 / 9$ (glasses) \\
2 & $6 / 18$ (glasses) & $6 / 9$ (glasses) \\
3 & CF (unaided)* & $6 / 9$ (glasses) \\
4 & $6 / 60$ (unaided)* & $6 / 12$ (glasses) \\
5 & $6 / 24$ (glasses) & $6 / 12$ (glasses) \\
6 & $6 / 36$ (unaided)* & $6 / 9$ (glasses) \\
7 & CF (unaided)* & $6 / 9$ (glasses) \\
\hline
\end{tabular}

GRS = graft refractive surgery; VA before GRS = acuity obtained with spectacle refraction as contact lens fitting was difficult because of the high cylinder.

*Refraction was difficult to perform because of the high cylinder.

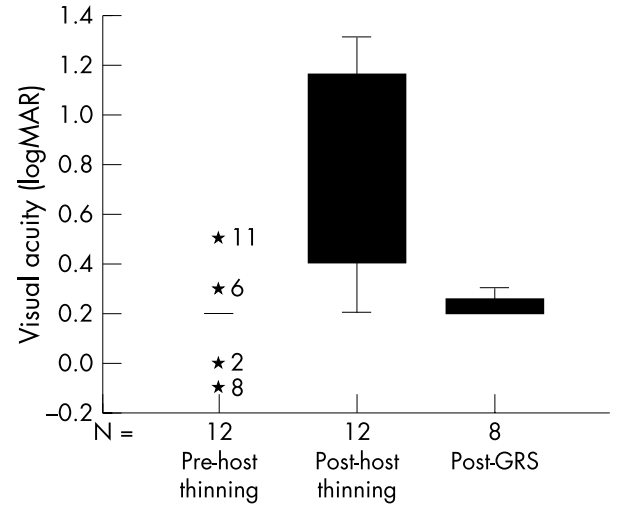

Figure 3 Box plots illustrating visual acuity before host thinning, after host thinning, and after graft refractive surgery (GRS).

ability with regard to axis. The mean was $-7.34^{\circ}\left(64.05^{\circ}\right)$ (clockwise).

\section{DISCUSSION}

Astigmatism after corneal transplantation is common. A subgroup of patients develops excessive astigmatism years after successful surgery. This group of patients has progression of ectasia in the recipient cornea, which is usually eccentric and creates attenuation of the wound in the affected area. This has a flattening effect on the donor cornea as the thinned host cornea weakens in its support for the donor cornea at the graft-host junction. This induced a steepening effect in the opposite meridian of the donor cornea with an average vector value of $7.59 \mathrm{D}$ in our series. The schematic diagram in figure 6 illustrates inferior host thinning with resultant weakening of the graft-host junction.

It is important to recognise patients in which this mechanism is present. Conventional approaches to postkeratoplasty astigmatism are unlikely to be effective. Incisional surgery in the steeper axis or LASIK (laser in situ keratomileusis) would be weakening procedures in an already ectatic eye. A "strengthening" procedure at the site of the problem, incision and suturing, or repeat keratoplasty would be more appropriate. Therefore, clinicians need to be aware of the pattern of disease resulting from progressive ectasia and be able to offer a rational corrective procedure based on an understanding of the pathophysiology.

These patients presented with decreasing visual acuity over a relatively short period of time (mean 10.2 months). This was coincident with increasing refractive and keratometric astigmatism. An area of host thinning was evident on slit lamp examination (fig l) in the area of host cornea with the flat meridian on keratometry. The mean disease induced astigmatism vector value was 7.59D. The direction of axis change did not show any recognisable trend. By giving the direction a sign based on the direction of the change and assuming random distribution of direction and magnitude of change, one would expect the derivation of a mean axis change to yield a value of zero. Here the value is $21^{\circ}$ in the anticlockwise direction but the numbers of eyes are small and the standard deviation is large (55.98).

There is a question as to whether larger PK needs to be performed for keratoconus in order to prevent or perhaps reduce the extent of late recurrence in the host cornea. However, it has been reported that larger grafts $(\geqslant 8.5 \mathrm{~mm})$ are associated with a higher failure rate compared to those of intermediate size $(>7.0 \mathrm{~mm}$ and $<8.5 \mathrm{~mm}) .{ }^{1}$ This may not be an acceptable risk given the low incidence of late progression in the host cornea. 


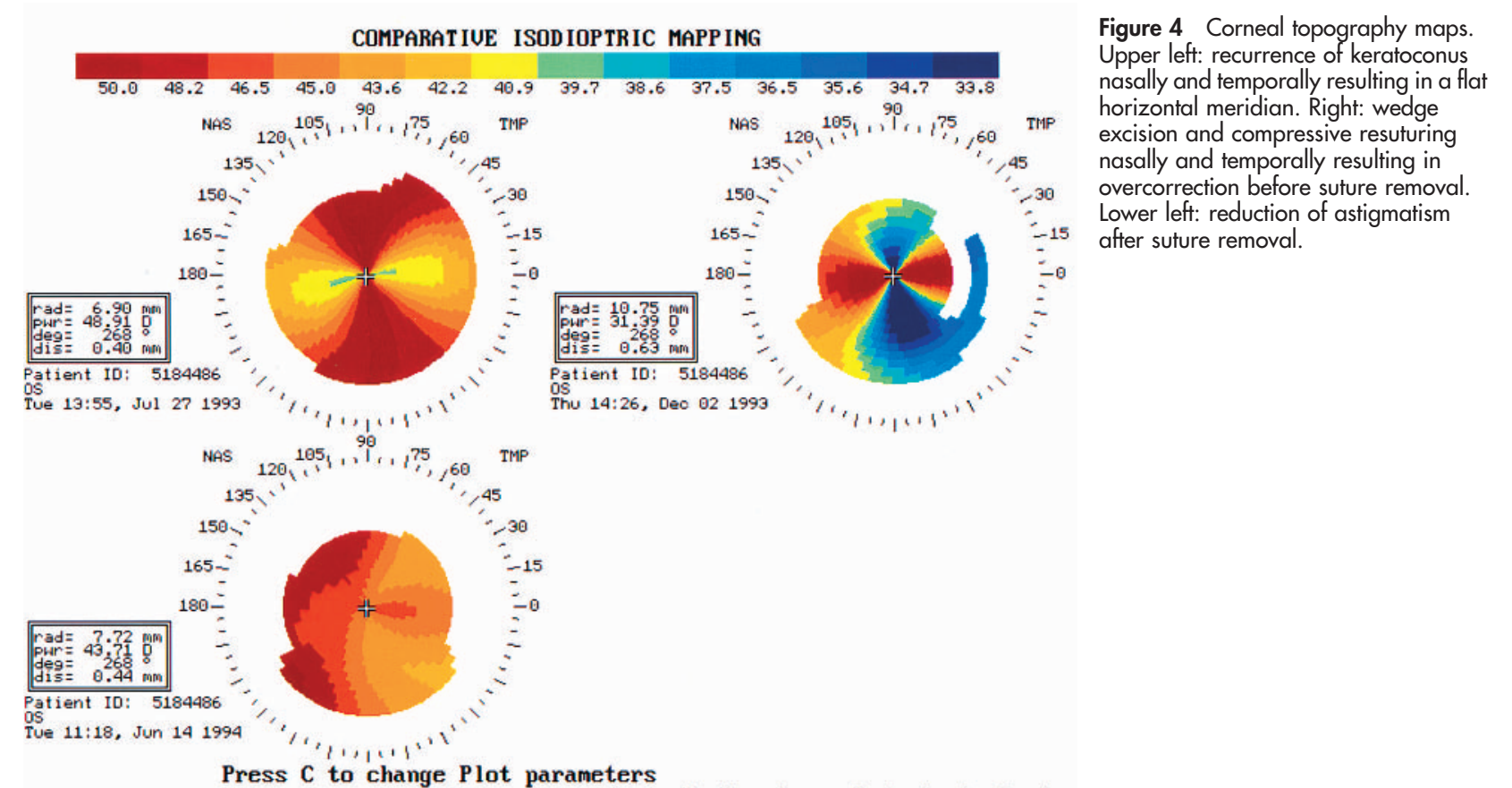

EyeSys Corneal Analysis System

These patients have an average of 11.00D of astigmatism, which makes correction with glasses or contact lenses difficult. Various surgical methods of correcting post-keratoplasty astigmatism (all sutures out astigmatism) have been described. These include relaxing incisions, compressive resuturing, relaxing incisions with counterquadrant augmenting compressive sutures (known as augmented relaxing incisions), wedge resections, trapezoidal keratectomy, and excimer laser refractive surgery. ${ }^{11-18}$ Trapezoidal keratectomies are technically difficult and the results are unpredictable. ${ }^{14}$ In our series of patients with high astigmatism resulting from host thinning we believe that the logical and best technique to employ would be compressive resuturing over the area of host thinning, that is, over the graft-host junction with the flat meridian. Although relaxing incisions along the steep meridian alone are appropriate for many cases of post-keratoplasty astigmatism, they will not improve the strength of the graft-host junction in the flat meridian, which is the cause of the astigmatism in host thinning (fig 6). It follows that it is important in cases of increased astigmatism in an old graft to examine the host cornea adjacent to the junction for any sign of thinning. For seven eyes, compressive resuturing was performed in the area with host thinning. The schematic diagram in figure 6 illustrates

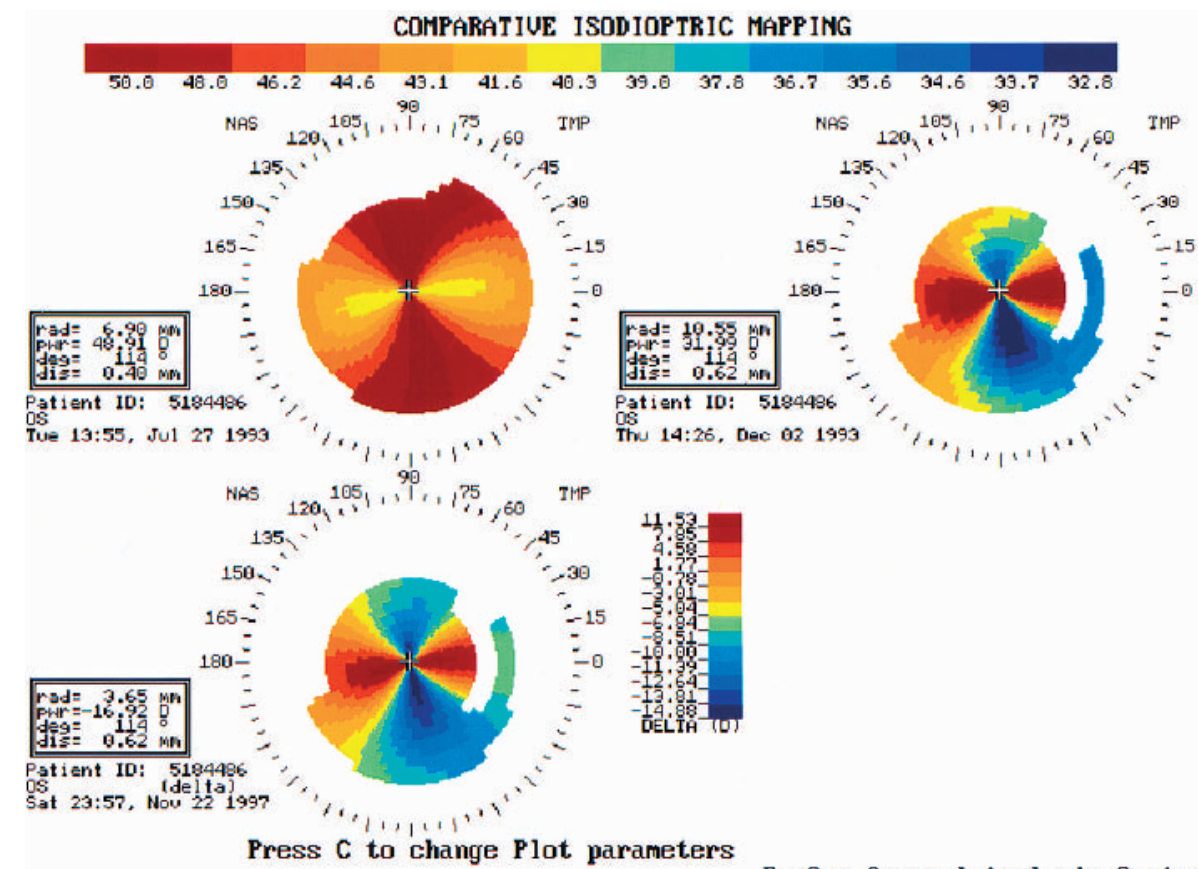

Figure 5 Corneal topography maps. Upper left: recurrence of keratoconus nasally and temporally resulting in a flat horizontal meridian. Right: wedge excision and compressive resuturing nasally and temporally resulting in overcorrection before suture removal. Lower left: difference map showing the surgical correction of astigmatism. 

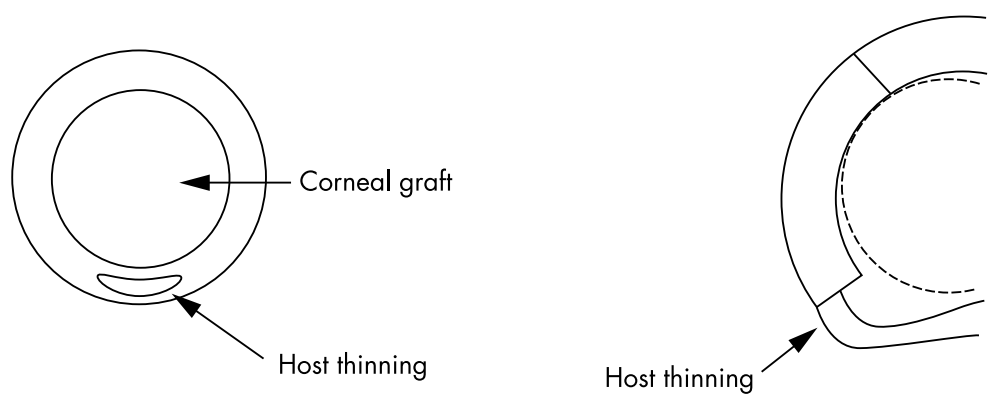

Figure 6 Schematic diagram illustrating: (Top) progression of keratoconus in the inferior host cornea with inferior host thinning resulting in weakening of the inferior graft-host junction. (Bottom) The technique of compressive resuturing over the grafthost junction with host thinning and resultant flat meridian as opposed to relaxing incisions over the graft-host junction with the steep meridian.

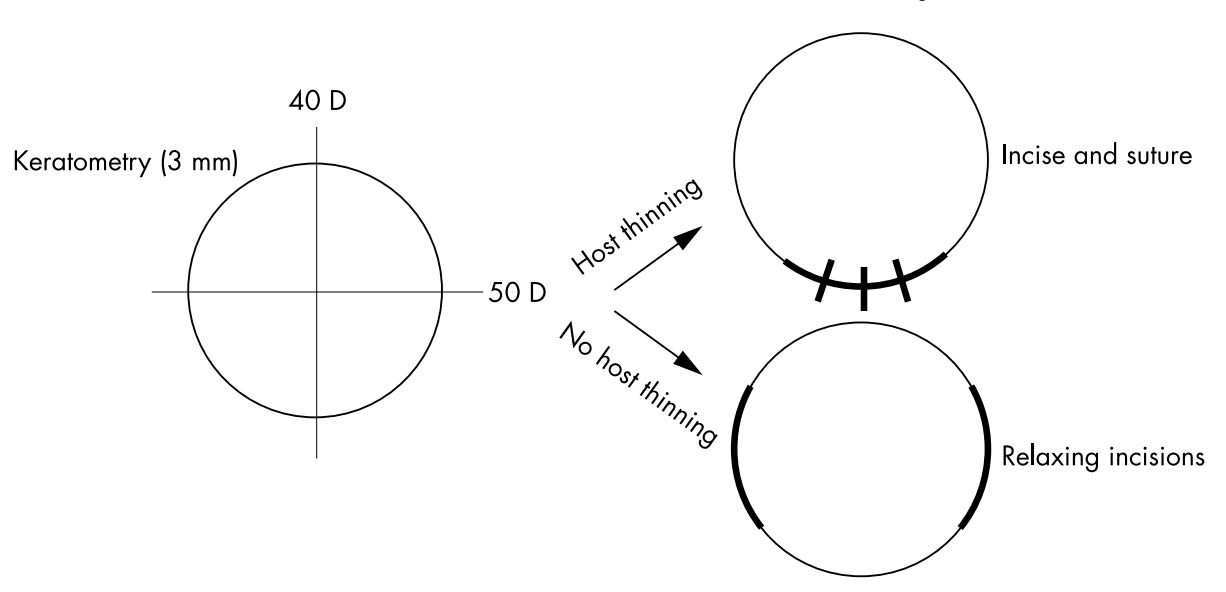

the theory behind our technique. If the host thinning results in more than 10D of astigmatism, wedge excision could be used in addition to compressive resuturing for more effect (patient 2).

This technique has resulted in the postoperative mean astigmatism of $7.09 \mathrm{D}$, which is a significant improvement from the preoperative mean astigmatism of $11.28 \mathrm{D}$ with a mean reduction of $4.2 \mathrm{D}$ of astigmatism by cylinder subtraction. This is comparable to previous reported studies on graft refractive surgery which reported a mean reduction in cylinder of 3.6-5D and 5.5D (for the group with preoperative astigmatism between 4-10D). ${ }^{15}{ }^{16}$ Although there appears to be little difference in the keratometric astigmatism before and after graft refractive surgery for patients 3, 5, and 7, these patients were able to tolerate corrective glasses after surgery. Perhaps the treatment of host thinning with compressive resuturing corrected some irregular astigmatism with resultant better spectacle tolerance.

The mean surgically induced astigmatism was just over 7D. It is notable that this is close to the mean disease induced vector value for the seven eyes undergoing graft refractive surgery $(8.42 \mathrm{D}(3.32))$. Taking a target value of $4 \mathrm{D}$ of remaining astigmatism, this was achieved, in vector terms, with a mean magnitude of error (SIA - TIA) of only 0.07D, with the SIA exceeding the TIA very slightly. Of course, in post-keratoplasty astigmatism the target of exactly $4 \mathrm{D}$, no more and no less, is simply a mathematical device to allow calculation of the success of the surgery. Here any value less than $4 \mathrm{D}$ is acceptable. This small mean magnitude of "error" shows us that this is a goal that may be reasonably achievable with this kind of surgery.

Mean postoperative remaining cylinder was approximately 7D. It appears inconsistent that a procedure that leaves a remaining cylinder mean power of $7 \mathrm{D}$, where $4 \mathrm{D}$ was the target, can be described as accurately achieving its aim. Using vector analysis, by combining the numerical descriptors of astigmatism (cylinder power and axis) in a unified mathematical expression, a vector with magnitude and direction, allows us to calculate astigmatic change in terms of power (magnitude) and axis (direction) where these two values vary in an interdependent way. Having done that, it then allows us to look at each of the two values independently. The apparent paradox of "excessive" remaining cylinder while describing the power change induced as "on target" is explained by the effect of misalignment of the treatment with the preoperative cylinder axis. The mean angle of error between SIA and TIA with the clockwise (minus) and anticlockwise (plus) convention described above was -7.34 . The fact that it was near zero implies an equal distribution of the surgically induced change about the preoperative axis. The wide standard deviation (plus or minus 64.05) is a measure of the relative unpredictability of the technique with regard to axis. Off axis treatments induce cylinder. There would appear to be little change in cylinder power in patients 3,5 , and 7 by cylinder subtraction. The low SIA values, by comparison with the TIA, for patients 5 and 7 indicate limited surgical effect, perhaps related to early suture removal in these cases (at 5 months versus a mean of 7.3 months in the other eyes). In case 3, however, the SIA exceeds the TIA yet leaves 14D of astigmatism. This is due to the "off-axis" effect of the surgery. The SIA orientation was at $3^{\circ}$ where the target was at $150^{\circ}$, dissipating the power effect and leaving only a change in axis.

In corneal wounds, the chord length, configuration, and distance of the wound from the visual axis determine its effect. Suturing the wound introduces another variable. In order to achieve the high power corrections targeted in this study several tight sutures must be placed. It is not surprising that the axis of the surgically induced astigmatism is frequently "misaligned." The length of the arc of graft-host junction incised is determined by the arc length affected by the disease, so restriction of incision length to allow more accurate induction of on-axis change is not possible, even if the power required were achievable with the few sutures that could be placed in a short wound.

In conclusion, patients presenting with increased astigmatism after PK should be examined for signs of host thinning in the flat meridian. Such cases can be satisfactorily managed 
with incision and compressive resuturing in the graft-host junction and, where astigmatism is high, additional wedge excisions.

\section{ACKNOWLEDGEMENTS}

Supported by the Ophthalmic Research Institute of Australia. KP is supported by NHMRC Sir Neil Hamilton Fairley Fellowship 0061. None of the authors has any proprietary interest related to this manuscript

\section{Authors' affiliations}

L Lim, K Pesudovs, M Goggin, D J Coster, Department of Ophthalmology, Flinders Medical Centre, Flinders University of South Australia, Bedford Park 5042, South Australia

\section{REFERENCES}

1 Williams KA, Muehlberg SM, Lewis RF, et al. The Australian Corneal Graft Registry, 1996 report

2 Kirkness $C M$, Ficker $L A$, Steele $A D$, et al. The success of penetrating keratoplasty for keratoconus. Eye 1990;4:673-88.

3 Lim L, Pesudovs K, Coster DJ. Penetrating keratoplasty for keratoconus: visual outcome and success. Ophthalmology 2000;107:1125-31.

4 Nirankari VS, Karesh J, Bastion F, et al. Recurrence of keratoconus in donor cornea 22 years after successful keratoplasty. Br J Ophthalmol 1983;67:23-8

5 Abelson MB, Collin HB, Gillette TE, et al. Recurrent keratoconus after keratoplasty. Am J Ophthalmol 1980;90:672-6.
6 Rubinfeld RS, Traboulsi El, Arentsen JJ, et al. Keratoconus after penetrating keratoplasty. Ophthalmic Surg 1990;21:420-2.

7 Bechrakis N, Blom ML, Stark WJ, et al. Recurrent keratoconus. Cornea 1994;13:73-7.

8 Kremer I, Eagle RC, Rapuano CJ, et al. Histologic evidence of recurrent keratoconus seven years after keratoplasty. Am J Ophthalmol 1995; 119:511-12.

9 Nordan NT. Keratoconus: Diagnosis and treatment. Int Ophthalmol Clin 1997;37:51-64.

10 Alpins NA. A new method of analysing vectors for changes in astigmatism. $J$ Cataract Refract Surg 1993;19:524-33.

11 Lindstrom RL, Lavery GW. Correction of postkeratoplasty astigmatism. In: Brightbill FS, ed. Corneal surgery, theory, technique, and tissue. St Louis: Mosby, 1986:333-43.

12 Lavery GW, Lindstrom RL, Hofer LA, et al. The surgical management of corneal astigmatism after penetrating keratoplasty. Ophthalmic Surg 1985;16:165-9.

13 Mandel MR, Shapiro MB, Krachmer JH. Relaxing incisions with augmentation sutures for the correction of postkeratoplasty astigmatism. Am J Ophthalmol 1987; 103:441-7.

14 Kelly CG. Surgical management of postkeratoplasty astigmatism. In: Kaufman HE, Barron BA, McDonald MB, Waltman SR, eds. The cornea. New York: Churchchill Livingstone, 1988:805-21.

15 Kirkness CM, Ficker LA, Steele AD, et al. Refractive surgery for graft-induced astigmatism after penetrating keratoplasty for keratoconus. Ophthalmology 1991;98:1786-92.

16 Fronterre A, Portesani GP. Relaxing incisions for postkeratoplasty astigmatism. Cornea 1991;10:305-11.

17 Campos M, Hertzog L, Garbus J, et al. Photorefractive keratectomy for severe postkeratoplasty astigmatism. Am J Ophthalmol 1992;114:429-36.

18 McCartney DL, Whitney CE, Stark WJ, et al. Refractive keratoplasty for disabling astigmatism after penetrating keratoplasty. Arch Ophthalmol 1987; 105:954-7. 